\title{
Descrição da Rede de Atenção à Saúde para o Controle do Câncer em Crianças e Adolescentes no Município do Rio de Janeiro
}

doi: https://doi.org/10.32635/2176-9745.RBC.2020v66n2.306

\author{
Description of the Health care Network for Cancer Control in Children and Adolescents in the City of Rio de Janeiro \\ Descripción de la Red de Atención a la Salud para Control del Cáncer en Niños y Adolescentes en el Municipio de Rio de \\ Janeiro
}

\author{
Monique Abreu Silvino'; Fabiano Saldanha Gomes de Oliveira ${ }^{2}$; Cid Manso de Mello Vianna ${ }^{3}$; Tania França ${ }^{4}$; Gerson Nunes da Cunha ${ }^{5}$
}

Resumo

Introduçáo: $\mathrm{O}$ câncer em crianças e adolescentes é a primeira causa de mortes por doença na faixa etária de 0-19 anos. O atraso na identificaçáo dos sinais e sintomas e a demora no encaminhamento das suspeitas diagnósticas podem alterar negativamente o prognóstico e serem responsáveis por um tratamento mais agressivo. Objetivo: Este estudo objetivou conhecer a estrutura, o funcionamento e os fluxos de acesso da Rede de Atençáo à Saúde (RAS) para o controle do câncer em crianças e adolescentes no município do Rio de Janeiro. Método: Trata-se de um estudo descritivo, que utilizou dados públicos de documentos, boletins on-line e dos sistemas de informaçóes, para descrever e mostrar a diagramação das informaçôes sobre essa RAS. Resultado: Os resultados mostram uma capacidade instalada que contempla as normativas, a existência de fluxos ordenados pela atenção primária em saúde, a regulação da assistência com oferta parcial da disponibilidade ambulatorial e hospitalar de diagnóstico e o tratamento do câncer infantojuvenil. Conclusáo: Esta pesquisa aponta a fragmentação dos sistemas de acesso dentro da RAS, a necessidade de atualização dos sistemas de informação relacionados ao câncer e indica que estudos de análise do cenário atual e a prospecção de cenários futuros podem melhorar o funcionamento atual, além de auxiliar na construção dos fluxos de saída dos hospitais habilitados.

Palavras-chave: Neoplasias; Crianças; Adolescentes; Serviços de Saúde; Gestão em Saúde.

\section{Abstract}

Introduction: Cancer in children and adolescents is the leading cause of deaths by disease in the 0-19 age group. Delayed identification of signs and symptoms and late referral of diagnostic suspicions may adversely affect the prognosis and be responsible for more aggressive treatment. Objective: This study aimed to know the structure, functioning and access flows of the health care network ( $\mathrm{HCN})$ for the control of cancer in children and adolescents in the city of Rio de Janeiro. Method: Descriptive study that used public documents, online bulletins and information systems to describe and show the logical structuring of information about this HCN. Results: The results show an installed capacity that includes the regulations, the existence of flows ordered by the Primary Health Care, the regulation of care with partial offer of outpatient and hospital availability of diagnosis and treatment of childhood and juvenile cancer. Conclusion: This research points out the fragmentation of the access systems within the HCN, the necessity to update the cancer-related information systems and indicates that studies of analysis and prospection of the future scenarios can improve the current functioning, besides helping to build the outflows of licensed hospitals. Key words: Neoplasms; Children; Adolescents; Health Services; Health Management.

\section{Resumen}

Introducción: El cáncer en nińos y adolescentes es la primera causa de muertes por enfermedad en el grupo de edad de 0-19 años. El retraso en la identificación de los signos y síntomas y demora en el encaminamiento de las sospechas diagnósticas pueden alterar negativamente el pronóstico y ser responsable de un tratamiento más agresivo. Objetivo: Este estudio objetiva conocer la estructura, el funcionamiento y flujos de acceso de la Red de Atención a la Salud (RAS) para controlar el cáncer en niños y adolescentes en el municipio de Río de Janeiro. Método: Se trata de un estudio descriptivo que utilizó datos públicos de documentos, boletines en línea y de los sistemas de información para describir y mostrar la diagramación de la información sobre esta RAS. Resultados: Los resultados muestran una capacidad instalada que contempla las normativas, la existencia de flujos ordenados por la Atención Primaria en Salud, la regulación de la asistencia con oferta parcial de la disponibilidad ambulatorial y hospitalaria de diagnóstico y tratamiento del cáncer infanto-juvenil. Conclusión: Esta investigación apunta fragmentación de los sistemas de acceso dentro de la RAS, necesidad de actualización de los sistemas de información relacionados al cáncer e indica que estudios de análisis del escenario actual y prospección de escenarios futuros pueden mejorar el funcionamiento actual además de auxiliar en la construcción de los flujos de salida de los hospitales habilitados. Palabras-clave: Neoplasias; Niño; Adolescente; Servicios de Salud; Gestión en Salud.

\footnotetext{
1 Seção de Oncologia Pediátrica do Instituto Nacional de Câncer José Gomes Alencar da Silva (INCA). Rio de Janeiro (RJ), Brasil. Orcid iD: https://orcid.org/ 0000-0002-2921-8738

${ }^{2}$ Departamento de Planejamento e Administração em Saúde do Instituto de Medicina Social da Universidade do Estado do Rio de Janeiro (IMS/Uerj). Rio de Janeiro (RJ), Brasil. Orcid iD: https://orcid.org/0000-0003-4121-0736

${ }_{3}^{3}$ Departamento de Planejamento e Administração em Saúde do IMS/Uerj. Rio de Janeiro (RJ), Brasil. Orcid iD: https://orcid.org/0000-0003-0252-1144

${ }_{4}^{4}$ Departamento de Planejamento e Administração em Saúde do IMS/Uerj. Rio de Janeiro (RJ), Brasil. Orcid iD: https://orcid.org/0000-0002-8209-9811

${ }^{5}$ Departamento de Informática da Fundação de Apoio à Escola Técnica (Faetec). Petrópolis (RJ), Brasil. Rio de Janeiro (RJ), Brasil. Orci iD: https://orcid.org/ 0000-0003-1127-8422

Endereço para correspondência: Monique Abreu Silvino. Rua Barão do Bom Retiro, 2563 - apto. 204 - Grajaú. Rio de Janeiro (RJ), Brasil. CEP $20540-341$. E-mail: masilvino@inca.gov.br
} 


\section{INTRODUÇÃO}

O câncer é raro na faixa etária infantojuvenil e seu tratamento exige estruturas físicas e equipamentos de alta complexidade e densidade tecnológica, além de uma equipe multiprofissional especializada ${ }^{1}$. O estudo da incidência, mortalidade e morbidade hospitalar na faixa etária de crianças, adolescentes e adultos jovens aponta um percentual mediano de neoplasias de $2 \%$ na populaçáo infantil (de 0 a 14 anos) e 3\% na populaçáo de crianças e adolescentes (de 0 a 19 anos), e mostra que os tumores infantojuvenis (de 0 a 19 anos) corresponderam a 2,8\% de todas as neoplasias informadas ${ }^{2}$.

Para que haja controle do câncer de crianças e adolescentes efetivo e em tempo oportuno, é necessário que, além do tratamento, outras modalidades de cuidado assistencial também sejam disponibilizadas e organizadas na Rede de Atenção à Saúde (RAS), destaca-se aqui a detecção precoce. O diagnóstico precoce conta com três etapas, sendo a primeira: a consciência dos sintomas do câncer, com acesso aos cuidados; a segunda: a avaliaçáo clínica, com diagnóstico e estadiamento; e a terceira: o acesso ao tratamento, incluindo alívio da dor ${ }^{3}$.

A Política Nacional para a Prevenção e Controle do Câncer na Rede de Atenção à Saúde das Pessoas com Doenças Crônicas, dada pela Portaria MS/GM n. ${ }^{\circ}$ 874 de $2013^{4}$, evidencia algumas responsabilidades e competências relacionadas aos pontos de atenção da rede. À atenção primária em saúde (APS), cabe a identificação de sinais e sintomas suspeitos dos tipos de cânceres.

Existem evidências de que crianças e adolescentes experimentam um tempo de diagnóstico prolongado, com grandes variaçôes entre os primeiros sintomas e o tempo gasto para investigar e tratar. Alguns fatores desse atraso estáo relacionados à incapacidade de comunicar sintomas, à consciência limitada e aos sintomas heterogêneos e não específicos que são semelhantes às condiçóes benignas ${ }^{3}$.

O Ministério da Saúde lançou uma publicação em 2017 intitulada "Protocolo de diagnóstico precoce para oncologia pediátrica" ${ }^{5 "}$, que busca auxiliar os profissionais da RAS a conduzir os casos suspeitos e confirmados dentro de uma linha de cuidado, mostrando as açôes desde a atenção básica até a alta complexidades. O município do Rio de Janeiro tem uma ação de promoção do diagnóstico precoce do câncer infantojuvenil, o Unidos pela Cura, que conta com a participação de gestores do Sistema Único de Saúde (SUS) nas três esferas, serviços especializados e da sociedade civil $^{6}$. Essa ação se integrou ao Plano Municipal de Saúde do Rio de Janeiro em 2010 e está presente no Plano de Atenção Oncológica do Estado, por meio da realização de capacitação dos profissionais da atençáo básica, a fim de possibilitar o diagnóstico precoce do câncer infantil.
Este estudo objetivou conhecer a estrutura, o funcionamento e os fluxos de acesso da RAS para o controle do câncer em crianças e adolescentes no município do Rio de Janeiro.

\section{MÉTODO}

Trata-se de um estudo transversal, com abordagem descritiva, desenvolvido por intermédio da análise documental, com levantamento de informações públicas do cenário de funcionamento da RAS para o controle do câncer em crianças e adolescentes do município do Rio de Janeiro. O fluxo de dados e processos foram descritos e posteriormente apresentados por uma ferramenta gráfica usada na análise de sistemas ${ }^{7}$. O diagrama de fluxo de dados, que organizou a fenomenologia das informaçóes encontradas em um modelo como representação abstrata de parte da realidade, pode ser usado para entender o seu funcionamento ${ }^{8}$.

Para elaboração dos diagramas de fluxos de dados, foram consultados os seguintes documentos: o Plano Municipal de Saúde (2010-2014) e (2014-2017) $)^{9,10}$; e o Plano Estadual de Atenção oncológica (2017) ${ }^{11}$. Também utilizaram-se as informaçôes das publicaçôes disponíveis on-line do Instituto Desiderata: Boletim ano 2, n. ${ }^{\circ} 2$, nov. 2015; Boletim ano 8, n. ${ }^{\circ}$ 14, maio 2016; Boletim ano 3, n. 3 , nov. 2016; Boletim informativo ano 9, ago. 2017; e Boletim vol. 4, n. ${ }^{\circ}$ 4, nov. $2017^{12-15}$.

A construção desse modelo também contou com levantamento de dados no Departamento de Informática do Sistema Único de Saúde (DATASUS), para obtenção de dados relativos à população. Do Cadastro Nacional de Estabelecimento de Saúde (CNES $)^{16}$ e da Plataforma da Subsecretaria de Atenção Primária, Vigilância e Promoção da Saúde (Subpav) ${ }^{17}$ do Rio de Janeiro, obtiveram-se dados sobre a estrutura física de atendimento.

Os dados disponíveis no Integrador $\mathrm{RHC}^{18}$ serviram como fonte de informação para a construção de fluxos, como os das Figuras 4 e 5, nos quais o Instituto Nacional de Câncer José Alencar Gomes da Silva (INCA) recebe crianças encaminhadas por outras fontes, além do Unidos pela Cura ou da Regulaçáo dos Serviços de Saúde no Rio de Janeiro (Reuni-RJ).

O Estudo segue as diretrizes da Resolução no ${ }^{\circ}$. 466/12 do Conselho Nacional de Saúde ${ }^{19}$ (CNS). Todos os dados e informaçôes utilizados nesta pesquisa são públicos e em sua maioria estão disponíveis para consulta on-line. Os dados disponibilizados por órgãos públicos e por organização não governamental são informaçôes que não envolvem o ser humano, de forma direta ou indireta, e foram cedidas mediante citação da fonte.

Os resultados apresentados também consideram referências importantes para atenção à saúde da criança 
e dos adolescentes como: a Política Nacional de Atenção Integral à Saúde da Criança; a Política Nacional de Atenção Integral à Saúde de Adolescentes e de Jovens; e o Estatuto da Criança e do Adolescente (ECA) ${ }^{20}$.

\section{RESULTADOS}

A partir das informaçóes encontradas nos documentos, foram feitas análises com desenvolvimento de diagramas seguidos da descrição textual, apresentando o segmento da RAS existente no município do Rio de Janeiro.

A Figura 1 condensa as informaçóes levantadas sobre a população pediátrica e a trajetória dentro da estrutura existente para atendimento de suspeitas de câncer infantojuvenil no município do Rio de Janeiro. Com destaque para a dificuldade na identificação única da população e na implantação do prontuário eletrônico nas unidades hospitalares e de atendimento de urgência.

A Figura 2 foi elaborada a partir da população, de suas faixas etárias e das informaçóes da estrutura de serviços, e considera a busca do usuário aos dois principais sistemas de acesso, e ainda mostra a interface da APS com o Unidos pela Cura, e da atenção secundária e terciária com a Reuni-RJ.

$\mathrm{Na}$ Figura 2, pode-se observar um encurtamento da trajetória quando o acesso é feito utilizando o fluxo da ação municipal do Unidos pela Cura. No entanto, a ação para diagnóstico precoce não abarca todos dos hospitais de diagnóstico de tratamento do câncer em crianças e adolescentes, deixando a oferta do recurso dividida entre os dois tipos principais de acessos: a Reuni-RJ e o Unidos pela Cura.

A Figura 3 detalha a interação da Reuni-RJ com os serviços de saúde da rede e mostra sua atuação como solicitante e executante da oferta de serviços ambulatoriais e de internação relacionados ao diagnóstico e ao tratamento do câncer em crianças e adolescentes no município do Rio de Janeiro. Salienta-se a presença das entidades externas que podem alterar o comportamento da RAS e o treinamento realizado pelo Unidos pela Cura. Percebe-se que, mesmo sendo a instituição da Política Nacional de Regulação do SUS de 2008, ainda há recurso especializado tanto ambulatorial quanto diagnóstico e terapêutico, que não está integralmente ofertado pela Reuni-RJ.

A Figura 4 mostra o diagrama que representa o Unidos pela Cura como processo dentro do sistema da RAS para o controle do câncer do município do Rio de Janeiro. Procurou-se esmiuçar o fluxo de encaminhamento, as açóes de treinamento tanto para APS quanto para Reuni-RJ e o sistema Unidos pela Cura utilizado pelas Coordenaçôes de Área Programática (CAP) como forma de agendamento eletrônico de consultas oncológicas em pediatria para esclarecimentos de suspeitas. O diagrama também exibe o acesso aos dados do sistema Unidos pela Cura feito pela Secretaria Municipal de Saúde para

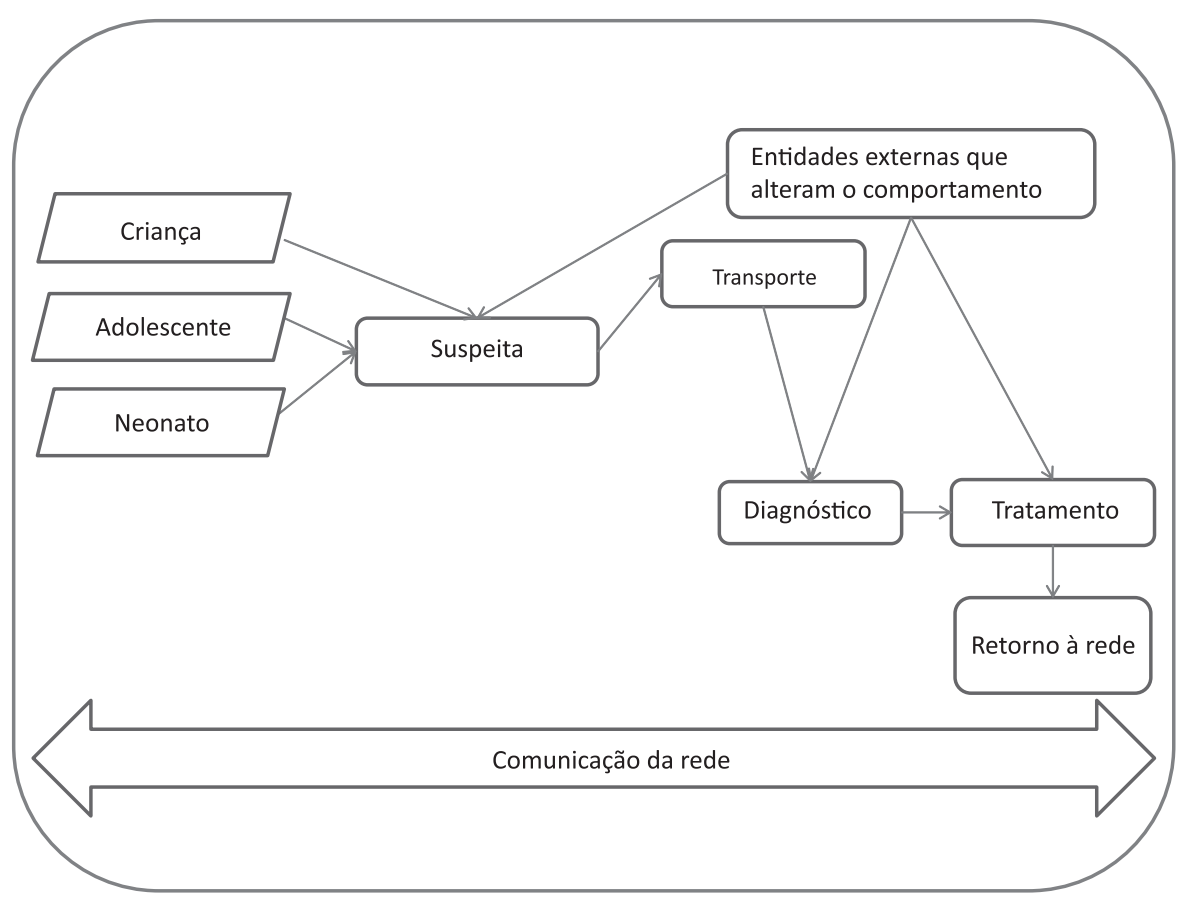

Figura 1. População e fluxo na estrutura de serviços na rede para controle do câncer do município do Rio de Janeiro 


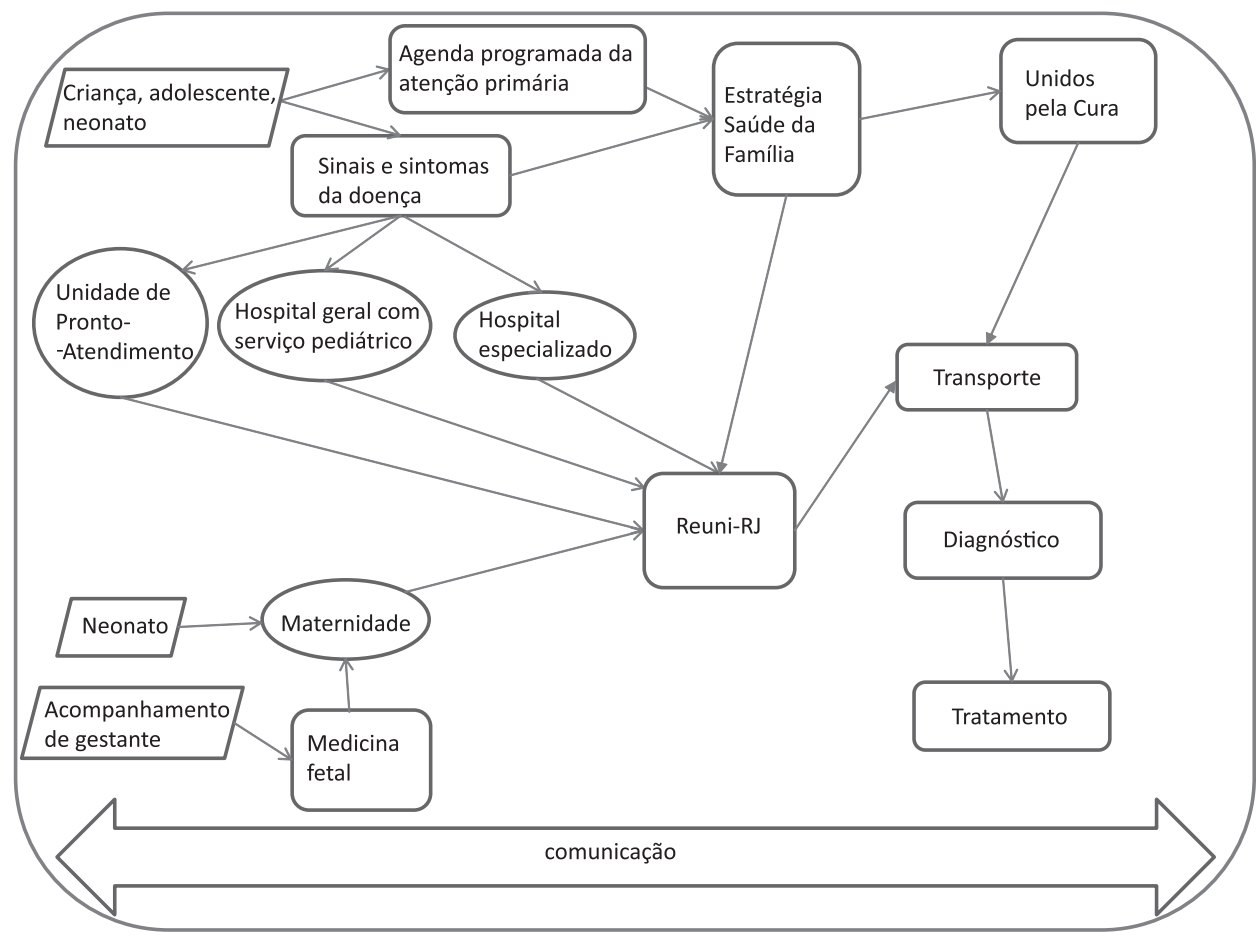

Figura 2. Diagrama da estrutura da rede para controle do câncer do município do Rio de Janeiro

Legenda: Reuni-RJ - Regulação dos Serviços de Saúde no Rio de Janeiro.

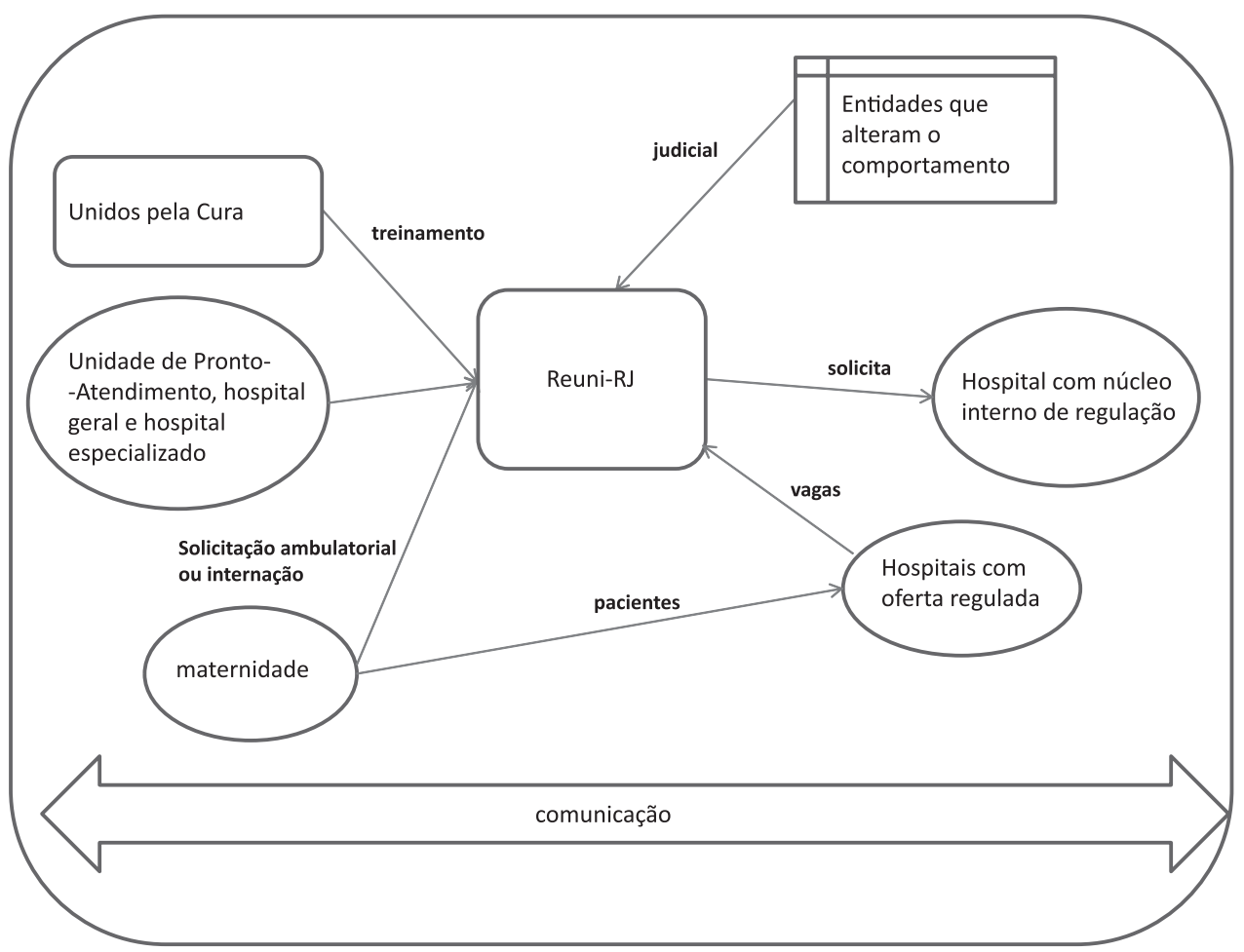

Figura 3. Diagrama da regulação da assistência da rede para controle do câncer no município do Rio de Janeiro

Legenda: Reuni-RJ - Regulação dos Serviços de Saúde no Rio de Janeiro. 


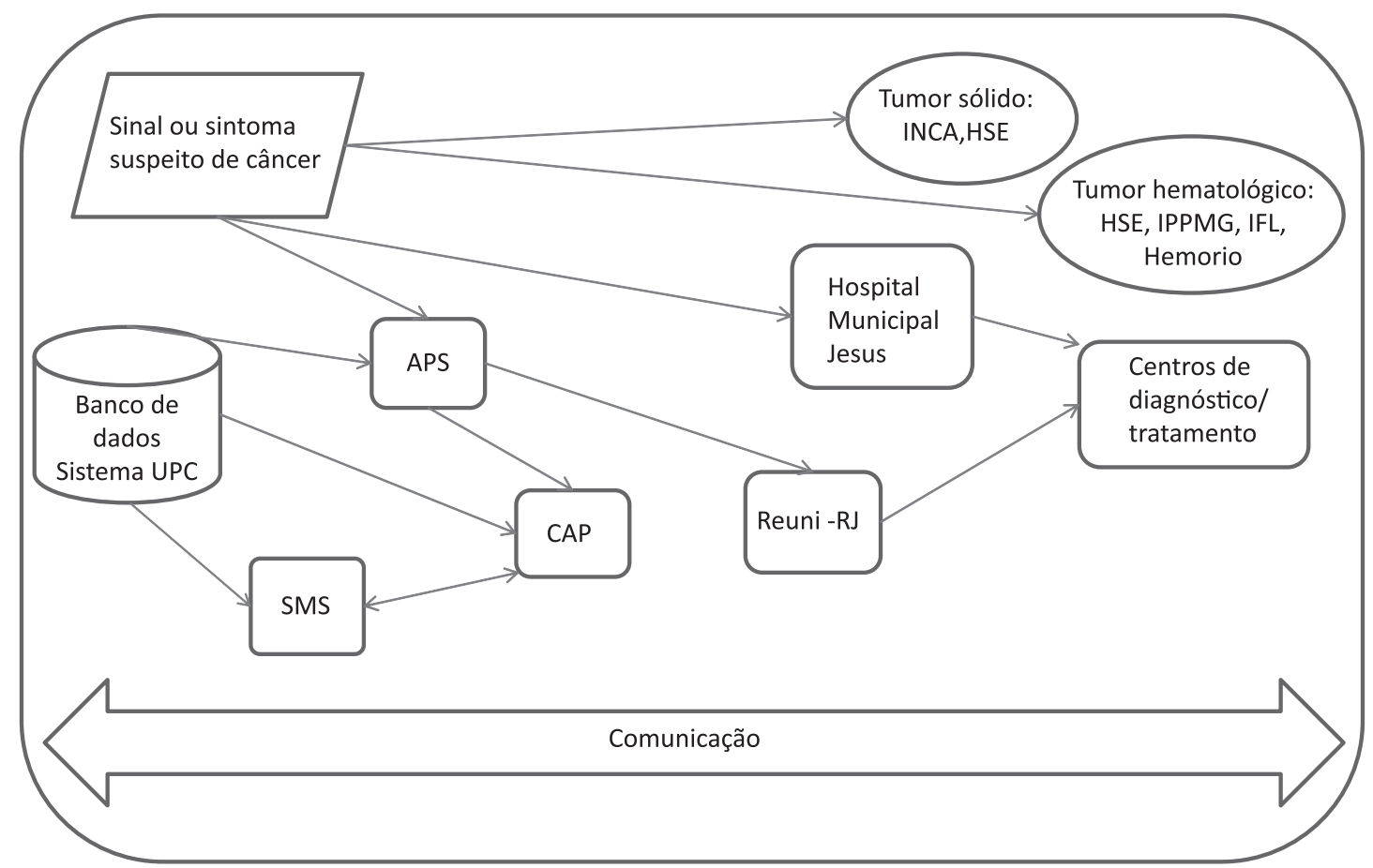

Figura 4. Diagrama da ação Unidos pela Cura na rede para controle do câncer do município do Rio de Janeiro

Legendas: APS - Atenção Primária em Saúde; CAP - Coordenação de Área Programática; IPPMG - Instituto de Puericultura e Pediatria Martagão Gesteira; IFL - Hospital Geral da Lagoa; INCA - Instituto Nacional de Câncer José Alencar Gomes da Silva; Hemorio - Instituto Estadual de Hematologia Arthur Siqueira Cavalcanti; HSE - Hospital dos Servidores do Estado; SMS - Secretaria Municipal de Saúde; UPC - Unidos pela Cura.

realização de busca ativa de crianças ou adolescentes que não compareceram ao agendamento da consulta, informação passada à APS que fica responsável pelo contato com a família e/ou responsável.

A Figura 5 foi elaborada a partir dos dados do Integrador RHC e representa o fluxo de crianças e adolescentes que realizam o tratamento oncológico na RAS do munícipio do Rio de Janeiro. Além do acesso pelo sistema Unidos pela Cura e pela Reuni-RJ, existem casos tratados em hospitais não habilitados, como Unidades ou Centros de Assistência de Alta Complexidade e Oncologia (Unacon/Cacon).

As informaçóes mostram o INCA como responsável por mais de $60 \%$ dos casos tratados no município do Rio de Janeiro. $\mathrm{O}$ acesso a esse Cacon habilitado com serviço de oncologia pediátrica também é feito via "Porta Aberta", termo utilizado pelo Instituto Desiderata.

$\mathrm{Na}$ Figura 5, é possível observar um fluxo de suspeitas que chega diretamente ao processo que representa o INCA e a expressividade numérica de casos tratados por esse Cacon é evidenciada pela espessura da seta que indica o fluxo.

É preciso construir fluxos específicos para os diagnósticos não confirmados que demandem um acompanhamento especializado ou tratamento por internação hospitalar nos serviços existentes na rede. A pactuação desse fluxo e a verificação dos serviços aptos a receber essa demanda também fazem parte do planejamento e organização dessa RAS.

Este estudo elaborou um diagrama de fluxo considerando algumas hipóteses de saída dos hospitais da rede de diagnóstico e tratamento do câncer infantojuvenil do Rio de Janeiro. A hipótese tem relação com as possibilidades de diagnóstico não maligno. Há questionamentos não respondidos por este estudo quanto à organização da RAS para o controle tardio do tratamento que, no diagrama, está simbolizado como ponto de interrogação.

\section{DISCUSSÃO}

Não está no escopo desta pesquisa, mas sabe-se que há aspectos sobre os fluxos entre as regióes de saúde atendidas pelo município dentro do Estado do Rio de Janeiro que devam ser comtemplados para suprir as necessidades de assistência oncológica pediátrica para a população.

O período de 2008 a 2018 sofreu um incremento de ações visando ao diagnóstico precoce do câncer infantojuvenil, despertando olhares dos pesquisadores. No entanto, observa-se um atraso na alimentação dos registros existentes que limita a elaboração de estudos com a utilização de dados recentes. Os achados evidenciam a necessidade de implantação do Registro de Câncer de Base Populacional (RCBP) ${ }^{21}$ no Estado do Rio de Janeiro para 


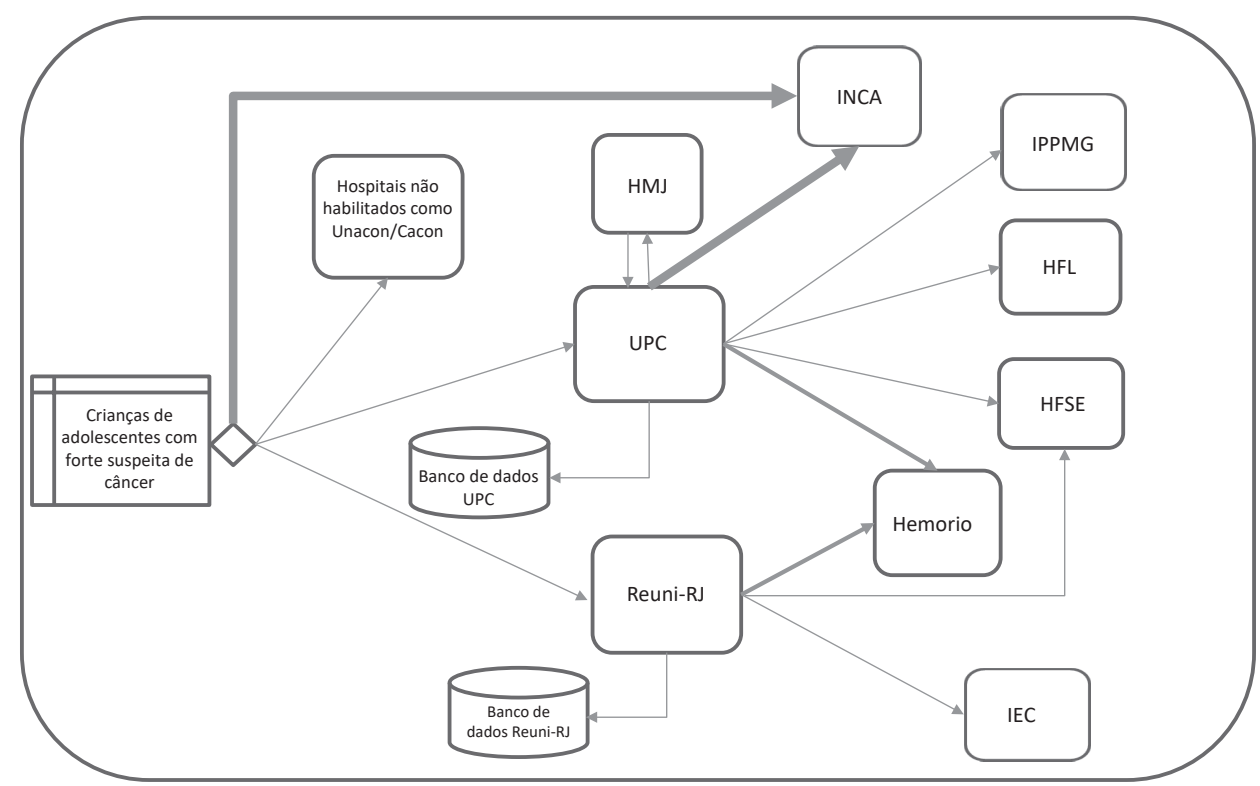

Figura 5. Diagrama do fluxo de tratamento de crianças e adolescentes na rede para controle do câncer do município do Rio de Janeiro

Legendas: IEC - Instituto Estadual do Cérebro Paulo Niemeyer; HFL - Hospital Federal da Lagoa; HFSE - Hospital dos Servidores do Estado; Hemorio - Instituto Estadual de Hematologia Arthur Siqueira Cavalcanti; INCA - Instituto Nacional de Câncer José Alencar Gomes da Silva; IEC - Hospital Estadual da Criança; HMJ - Hospital Municipal Jesus; UPC - Unidos pela Cura; IPPMG - Instituto de Puericultura e Pediatria Martagão Gesteira; Reuni-RJ - Regulação dos Serviços de Saúde no Rio de Janeiro.

que a gestão e o planejamento das açóes de saúde nessa RAS sejam elaborados adequadamente.

A comunicação entre os serviços da RAS não privilegia a gestão assistencial, dada a modalidade informal utilizada. Há problemas encontrados na implantação dos identificadores únicos e uma desigualdade na cobertura de utilização de informação eletrônica clínica nos diferentes níveis de atenção.

O encaminhamento via regulação da assistência ou via cartão Unidos pela Cura náo oferece a possibilidade de uma comunicaçáo efetiva entre o ponto de encaminhamento da RAS e os centros de alta complexidade para realizar alerta de casos de baixa suspeitas de câncer infantojuvenil. Visando ao fortalecimento e à resolutividade da RAS, é preciso pensar nas possibilidades de comunicação existentes para chamar atenção dos encaminhamentos dos casos de baixa suspeita para centro de alta complexidade.

No que se refere ao acesso, o estudo mostra que há um processo ligado à Reuni-RJ e outro ligado ao Unidos pela Cura e que ambos náo ofertam a totalidade da capacidade de diagnóstico e tratamento do câncer infantojuvenil do município. Isto parece reforçar a histórica fragmentação do sistema de saúde.

Para o ano de 2018, no Plano Estadual Oncológico, foi prevista uma identificação dos problemas apresentados pelas unidades habilitadas com baixa produção em cada modalidade de atenção. Para os casos pediátricos, alguns dos problemas são velhos conhecidos e estão relacionados à estrutura das unidades (limitação da produção por ausência de leito de terapia intensiva no estabelecimento de saúde é um exemplo). A estrutura física e o mobiliário existente impedem a ampliação da faixa etária de atendimento e a disponibilidade de uma equipe multidisciplinar completa ou em quantidade adequada para atendimento.

A crescente importância dos medicamentos na atenção à saúde, sua desigualdade de acesso, ciclos logísticos e a interface com a gestão clínica para o atendimento das necessidades de saúde de uma populaçáo, bem como os processos que envolvem a aquisiçấo de medicamentos e insumos e sua relação com a disponibilidade orçamentária e financeira em presença das influências do poder judiciário, deslocam os fluxos e também podem ser representados por diagramas.

Aponta-se a necessidade de opçóes estratégicas que Godet $^{22}$ demarca como "a ambição da vontade se inscreve no princípio da realidade das evoluçóes previsíveis (...)" e de prospecção de cenários "que projectam os desejos e as angústias face ao futuro". Isto é, pensar nas mudanças avaliando as opçóes estratégicas possíveis, elaborando planos de organização para se preparar para as mudanças esperadas, provocando as mudanças desejadas.

\section{CONCLUSÃO}

Entende-se que é preciso pensar no desenvolvimento de estudos prospectivos do cenário para melhorias da 
RAS em questão, visando a testes simulados de cenários de fluxos para acolhimento dos diagnósticos diferenciais, modificaçóes dos perfis de atendimento dos hospitais, alteraçóes possíveis nos sistemas de acesso entre outras.

$\mathrm{O}$ avanço ou progresso ou melhoria da capacidade dos computadores e das técnicas de simulaçôes computacionais permitem o uso do método como ferramenta de planejamento e de suporte à decisão, utilizando um número significativo de variáveis concomitantes em um ambiente controlado. Este estudo mostra o caminho para construção de um modelo de simulação da fenomenologia desse cenário e busca futuramente utilizar essa ferramenta com propósito de testar estratégias e açóes que contribuam para o controle do câncer em crianças e adolescentes.

\section{CONTRIBUIÇÕES}

Monique Abreu Silvino e Fabiano Saldanha Gomes de Oliveira Engenheiro participaram da concepção ou desenho do trabalho; aquisição, análise e ou interpretação dos dados de pesquisa. Cid Manso de Mello Vianna, Tania França e Gerson Nunes da Cunha participaram da redaçáo e/ou revisão crítica com contribuição intelectual. Todos os autores aprovaram a versão final a ser publicada.

\section{AGRADECIMENTOS}

À Divisão de Detecção Precoce e Apoio à Organização de Rede do INCA pela cessão dos dados das Autorizaçóes de Procedimentos de Alta Complexidade de 2015.

\section{DECLARAÇÃO DE CONFLITO DE INTERESSES}

Nada a declarar

\section{FONTES DE FINANCIAMENTO}

Não há.

\section{REFERÊNCIAS}

1. Instituto Nacional de Câncer José Alencar Gomes da Silva. Diagnóstico precoce do câncer na criança e no adolescente [Internet]. 2 ed. rev. ampl. Rio de Janeiro: INCA; 2014. [acesso 2017 fev 04]. Disponível em: http://www1.inca.gov.br/inca/Arquivos/protocolo-dediagnostico-precoce-do-cancer-pediatrico.pdf

2. Instituto Nacional de Câncer José Alencar Gomes da Silva. Incidência, mortalidade e morbidade hospitalar por câncer em crianças, adolescentes e adultos jovens no Brasil: informaçóes dos registros de câncer e do sistema de mortalidade [Internet]. Rio de Janeiro: INCA; 2016. [acesso 2017 ago 16]. Disponível em: http://www1.inca. gov.br/wcm/incidencia/2017/pdf/versao-completa.pdf/
3. World Health Organization. Guide to cancer early diagnosis [Internet]. Geneva: WHO; 2017. [cited 2017 Sept 06]. Available from: https://www.who.int/cancer/ publications/cancer_early_diagnosis/en/

4. Ministério da Saúde (BR), Gabinete do Ministro. Portaria $\mathrm{n}^{\circ}$ 874, de 16 de maio de 2013. Institui a Política Nacional para a Prevençáo e Controle do Câncer na Rede de Atenção à Saúde das Pessoas com Doenças Crônicas no âmbito do Sistema Único de Saúde (SUS). Diário Oficial da União, Brasília, DF; 2013 maio 17. Seção I, p. 129.

5. Ministério da Saúde (BR). Protocolo de diagnóstico precoce para oncologia pediátrica [Internet]. Brasília, DF: Ministério da Saúde; 2017 [acesso 2018 fev 17]. Disponível em: http://bvsms.saude.gov.br/bvs/ publicacoes/protocolo_diagnostico_precoce_cancer_ pediatrico.pdf

6. Silva MAV. Câncer infanto juvenil trajetória dos casos com suspeição realizada em unidades não especializadas no município do Rio de Janeiro [dissertação na Internet]. Rio de Janeiro: Instituto Nacional de Saúde da Mulher, da Criança e do Adolescente Fernandes Figueira; 2011 [acesso 2017 fev 12]. Disponível em: https://www.arca. fiocruz.br/bitstream/icict/7914/2/69164.pdf

7. Gane C, Sarson T. Análise estruturada de sistemas. Rio de Janeiro: Livros Técnicos e Científicos;1983. 257 p. (Série aplicaçóes de computadores).

8. Borshchev A. The big book of simulation modeling: multimethod modeling with anylogic 6. Chicago: AnyLogic América do Norte; 2013.

9. Secretaria Municipal de Saúde e Defesa Civil (RJ). Plano municipal de saúde do Rio de Janeiro: PMS 2010-2013 [Internet]. Rio de Janeiro: SMSDC; 2009 [acesso 2017 mar 03]. Disponível em: http:// www.rio.rj.gov.br/dlstatic/10112/3700816/4130215/ PLANOMUNICIPALDESAUDE20102013.pdf

10. Secretaria Municipal de Saúde e Defesa Civil (RJ). Plano municipal de saúde do Rio de Janeiro: PMS 2014-2017 [Internet]. Rio de Janeiro: SMSDC; 2013 [acesso 2017 mar 04]. Disponível em: http:// www.rio.rj.gov.br/dlstatic/10112/3700816/4128745/ PMS_20142017.pdf

11. Secretaria de Saúde (RJ). Plano estadual de atenção oncológica [Internet]. Rio de Janeiro: Impr. Oficial do Estado; 2017 maio [acesso 2017 out 02]. Disponível em: http://www.cib.rj.gov.br/arquivos-para-baixar/boletinscib/2228-planoatencaooncologicafinal-centrosregionais diagnostico-052017/file.html

12. Instituto Desiderata. Boletim Informativo Unidos pela Cura [Internet]. Rio de Janeiro: Desiderata. Ano 7, No. 13, maio 2015 [acesso 2017 maio 07]. Disponível em: http://www.informacaoemsaude.rj.gov.br/docman/ informes-ao-gestor/11482-boletim-com-link-parapesquisa-desiderata.html 
13. Instituto Desiderata I. Panorama da oncologia pediátrica [Internet]. Rio de Janeiro: Desiderata. Ano 3, No. 3, nov 2016 [acesso 2017 mar 16]. Disponível em: http://desiderata.dev.tuut.com.br/wp-content/ uploads/2019/01/boletim_panorama_2016_web.pdf

14. Instituto Desiderata. Boletim Informativo Unidos pela Cura [Internet]. Rio de Janeiro: Desiderata. Ano 8, No. 14, maio 2016 [acesso 2017 mar 16]. Disponível em: https://desiderata.org.br/ra2015/assets/@ boletim14_11mai2016.pdf

15. Instituto Desiderata. Panorama da oncologia pediátrica [Internet]. Rio de Janeiro: Desiderata. Ano 4, No. 4, nov 2017 [acesso 2017 mar 16]. Disponível em: http://desiderata.dev.tuut.com.br/wp-content/ uploads/2019/01/boletim_panorama_2017_web.pdf

16. Ministério da Saúde (BR), Departamento de Informática do Sistema Único de Saúde. Cadastro nacional de estabelecimento de saúde [Internet]. Brasília, DF: DATASUS; [2017] [acesso 2017 fev 21]. Disponível em: http://cnes.datasus.gov.br/

17. Prefeitura do Rio de Janeiro. Plataforma Subpav [Internet]. Rio de Janeiro: Equipe do Núcleo de Informaçóes da SAP; 2016 [acesso 2017 abr 14]. Disponível em: https://subpav.org/logar.php

18. Instituto Nacional de Câncer José Alencar Gomes da Silva. IntegradorRHC [Internet]. Versão 3.2. Rio de Janeiro: INCA [data desconhecida] - [atualizada 2012 dez 14; acesso 2017 abr 14]. Disponível em: https://irhc. inca.gov.br/RHCNet/

19. Conselho Nacional de Saúde (BR). Resolução no 466, de 12 de dezembro de 2012 [Internet]. [acesso 2017 Ago 30]. Disponível em: http://conselho.saude.gov.br/ resolucoes/2012/Reso466.pdf

20. Congresso Nacional (BR), Senado Federal. Estatuto da criança e do adolescente [Internet]. Brasília, DF: Senado Federal; 2017 [acesso 2017 nov 20]. Disponível em: http://www2.senado.leg.br/bdsf/bitstream/handle/ id/534718/eca_1ed.pdf

21. Instituto Nacional de Câncer José Alencar Gomes da Silva. Manual de rotinas e procedimentos para registro de câncer de base Populacional [Internet]. 2 ed. rev. atual. Rio de Janeiro: INCA; 2012 [acesso 2017 maio 15]. Disponível em: https://www.inca.gov.br/sites/ ufu.sti.inca.local/files//media/document//manual-derotinas-e-procedimentos-para-registros-de-cancer-debase-populacional.pdf

22. Godet M. A "Caixa de Ferramentas" da prospectiva estratégica [Internet]. Lisboa: CEPES; [2000] [acesso 2017 nov 22]. Disponível em: http://www.institutobrasilrural. org.br/download/20080615095245.pdf 